\title{
Il peso della storia, la voce della tragedia. II lavoro teatrale di Archivio Zeta nei paesaggi del Novecento
}

L'articolo presenta il lavoro teatrale dell'associazione culturale Archivio Zeta, che ha per scenario una serie di luoghi storici di Bologna e dintorni.

The article presents the theatrical work of the cultural association Archivio Zeta, staged in some historical sites in Bologna and its environs.

\section{Teatro e difficult heritage}

Salendo sull'Appennino tra Bologna e Firenze, nei pressi del passo della Futa, si scorge in mezzo ai boschi una sorta di alta lama di pietra. Si tratta del coronamento scultoreo del Deutscher Soldatenfriedhof Futapass, il cimitero militare germanico, costruito tra il 1961 e il 1969 su progetto dell'architetto Dieter Oesterlen. Qui vennero raccolte 30.683 salme di soldati tedeschi morti durante il secondo conflitto mondiale e sepolti in diverse località d'Italia: alla metà degli anni Cinquanta venne stipulato un accordo tra i due paesi affinché venissero ceduti i terreni gratuitamente, mentre la loro gestione sarebbe stata affidata al Volksbund Deutsche Kriegsgräberfürsorge, l'ente privato creato nel 1919 e sovvenzionato dallo stato tedesco.

Tuttavia, negli anni Sessanta un cimitero di guerra tedesco, in terra italiana, anzi, sulla dorsale appenninica dove passava la linea gotica, non poteva essere disegnato attingendo alle forme monumentali che avevano caratterizzato questi sa- 
crari prima di quel conflitto. Oesterlen lo concepì quindi come un intervento di architettura nel paesaggio: la cima della collina viene avvolta da un muro continuo in blocchi di arenaria scura, arabescata nel tempo da licheni gialli, che sale a spirale creando una serie di terrazze dove, a terra, sono posate le lapidi. Il culmine di questo movimento ascensionale è la lama svettante verso il cielo.

Non sono molti i visitatori che si recano in questo luogo: alcuni gruppi legati ad associazioni tedesche, qualche campeggiatore di passaggio. Tuttavia, negli ultimi quindici anni, nelle due settimane centrali di agosto, ogni pomeriggio poco prima del tramonto, salgono lassù alcune centinaia di persone, per lo più da Firenze $\mathrm{e}$ da Bologna. A partire dal 2003, infatti, il cimitero militare tedesco al Passo della

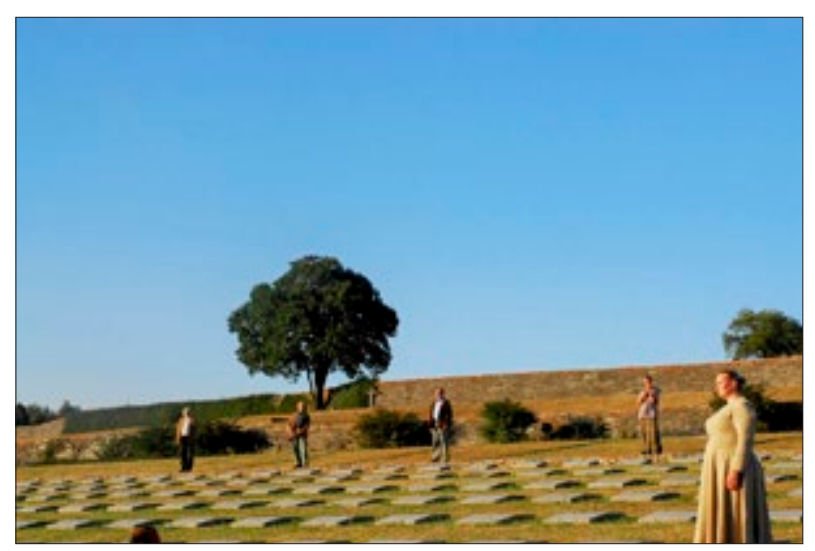

Fig. 1. I Persiani di Eschilo, Archivio Zeta al Cimitero militare germanico al passo della Futa, 2003 (foto: Franco Guardascione).

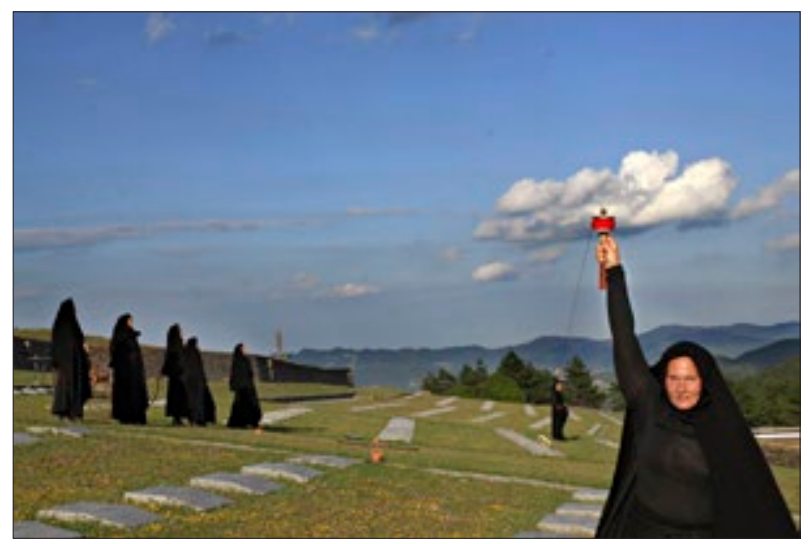

Fig. 2. Coefore di Eschilo, Archivio Zeta al Cimitero militare germanico al Passo della Futa, 2011 (foto: Franco Guardascione)
Futa è diventato lo spazio scenico per i progetti teatrali della compagnia Archivio Zeta.

La prima opera a essere messa in scena lassù fu I persiani di Eschilo, cui seguirono Sette contro Tebe, poi l'Antigone di Sofocle, e ancora l'Iliade, l'intero ciclo dell'Orestea, Gli ultimi giorni dell'umanità di Karl Kraus, il Macbeth di Shakespeare, mentre quest'estate sarà la volta de $I l$ Minotauro di Cortázar. Il muro del cimitero della Futa è stato di volta in volta fortificazione di Troia, cinta di Tebe, trincea della Grande guerra, castello scozzese avvolto dalla foresta che si muove, e presto sarà il labirinto di Cnosso. Ma soprattutto ha continuato a essere quello che è, ovve- 
ro il luogo ultimo dei nemici. Quale luogo migliore, per la messa in scena di una tragedia, che il luogo della memoria di un conflitto? E della memoria più difficile, quella dei perpetratori, morti, uccisi come le loro vittime.

Nell'intero lavoro teatrale di Archivio Zeta il luogo svolge un ruolo fondamentale, divenendo palcoscenico, scenografia, quadro e contesto dell'azione. Da molti anni, infatti, Enrica Sangiovanni e Gianluca Guidotti hanno prediletto per le loro trasposizioni luoghi significativi, immersi nel paesaggio naturale o urbano, carichi di una stratigrafia di storie, spesso faticosi, irti, spazzati dal vento, difficili da raggiungere o gravati da un difficult heritage, un peso storico arduo da portare.

\section{Il progetto Pilade/Pasolini tra Monte Sole, Volterra e Bologna}

Nel 2015 Archivio Zeta ha dedicato un anno di lavoro al Pilade di Pier Paolo Pasolini, un testo moderno costruito attorno a una figura, Pilade, che nell'Orestea pronuncia una sola battuta. In quel caso, Sangiovanni e Guidotti hanno scelto di portare le parole di Pasolini in luoghi a lui affini, legati ai suoi temi più cari: i luoghi della Resistenza, quelli della periferia in cambiamento, ma anche i luoghi del lavoro e i centri del potere.

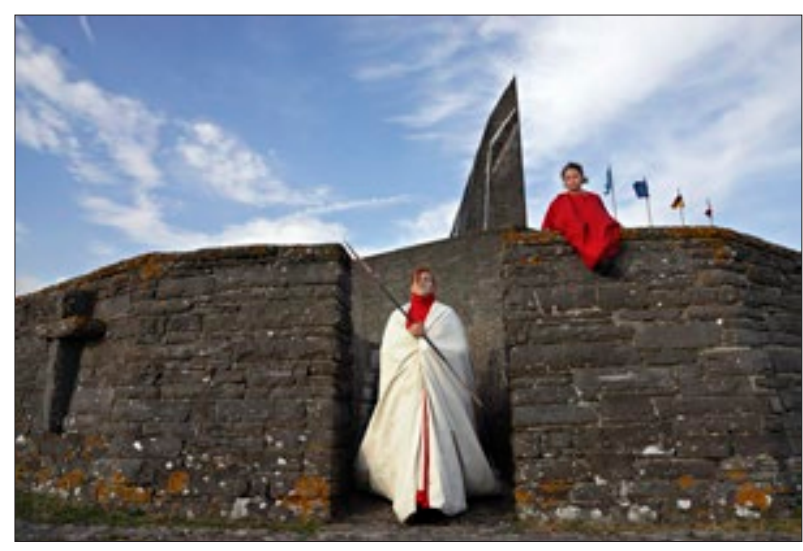

Fig.3. Pilade di Pasolini, Archivio Zeta al Cimitero militare germanico al Passo della Futa, 2015 (foto: Franco Guardascione).

Smontando il testo e privandolo della sua sequenzialità per soffermarsi su ogni singola parte, il primo frammento Pilade/Montagne è stato messo in scena tra i boschi, i prati e le rovine di Monte Sole, nei luoghi degli eccidi dell'autunno 1944, in occasione del settantesimo della Liberazione, il 25 aprile. "Le montagne saranno cumuli di gioventù silenziosa. / La pianura un formicolare di madri con poveri lumi”: nel Pilade sono le Eumenidi a pronunciare queste parole, sotto forma di rivelazione. 
Nell'estate del 2015, gli altri frammenti della "tragica analisi" di Pasolini sono stati messi in scena a Volterra, dove, ad esempio, la nascita di Atena è avvenuta tra le fumarole di Sasso Pisano, il Campo dei Rivoluzionari è stato ricreato in un luogo del lavoro come le Saline.

Il $1^{\circ}$ novembre il Pilade "a pezzi" è stato ricomposto e portato a Bologna, città in cui Pasolini nacque e dove tornò in seguito per studiare. In tre tempi, il testo è stato messo in scena in una "maratona" attorno al pronao di Villa Aldini, una villa neoclassica incompiuta, poi al poligono di tiro e infine sotto alla pensilina dell'ex mercato ortofrutticolo: tutti luoghi periferici rispetto al cuore della città, che continua a restare quello cinto da mura abbattute più di un secolo fa. Luoghi periferici, luoghi in trasformazione, luoghi che non fanno parte dei percorsi quotidiani dei cittadini. E luoghi legati alla storia della città, più o meno recente. Villa Aldini si trova sulla collina che domina Bologna, nel punto dove Napoleone godette della vista sulla città; i suoi lavori iniziarono nel 1811 ma non riuscirono a essere completati prima della sconfitta di Bonaparte. Dopo avere cambiato molte funzioni, negli anni Trenta divenne Monumento alla Vittoria e le fu accostato un edificio destinato a ospitare i familiari dei caduti nella Grande guerra. Successivamente divenne una casa di riposo, sempre con un'attenzione particolare per chi aveva sofferto la guerra, non più la prima, ma la seconda. È alla metà degli anni Settanta che Pasolini scelse questo luogo come set per gli esterni della "villa dei

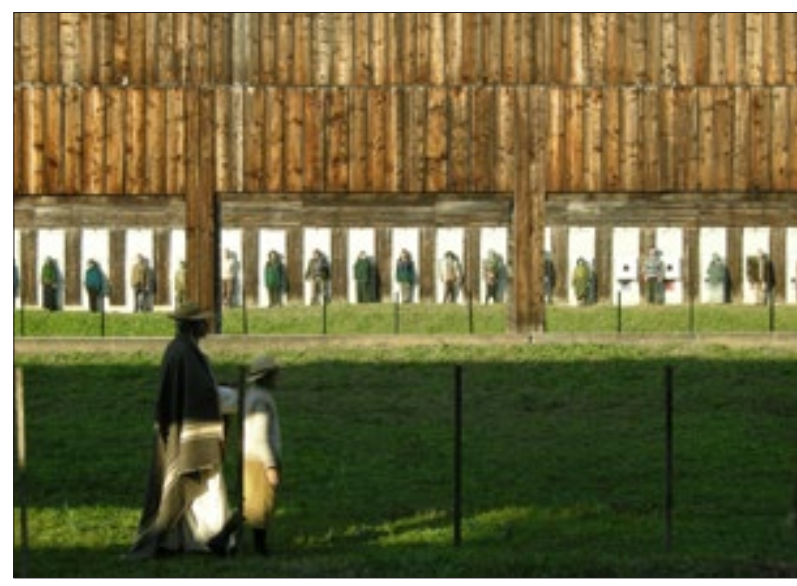

Fig. 4. Pilade di Pasolini, Archivio Zeta al poligono di tiro di Bologna, 2015 (foto: Elena Pirazzoli).
Signori" in Salò o le 120 giornate di Sodoma, dove vengono lette le "regole" da quello stesso pronao. Dal 2014 l'edificio della casa di riposo accoglie richiedenti asilo: per lo più giovani uomini, fuggiti da guerre e massacri e recuperati nel Mediterraneo con l'operazione Mare Nostrum, ora in attesa di conoscere l'esito della domanda di protezione internazionale.

Il poligono di tiro, costruito nei primi anni del Novecento, venne utilizzato dalla Repubblica sociale italiana per le esecuzioni dei partigiani: la memoria di quelle esecuzioni in un luogo 
appartato e recintato non è viva come per quelle effettuate in piazza Nettuno, dove venne creato spontaneamente, il giorno della Liberazione di Bologna, un sacrario fatto di fotografie estratte dai portafogli o tolte dai comodini o dai muri delle sale da pranzo. Un monumento e una lapide sul lato del complesso lo ricordano oggi, mentre al suo interno continuano a svolgersi gare e corsi di tiro.

Infine, l'ex mercato ortofrutticolo è stato oggetto negli ultimi anni di un ampio progetto di trasformazione urbana: sono stati abbattuti quasi tutti i vecchi edifici e impianti, a parte l'ingresso monumentale di carattere razionalista e la pensilina attribuita a Pierluigi Nervi (ma in realtà di Renato Bernardi). Su quello spazio è in corso da anni un cantiere per la costruzione di un complesso di edifici a torre a destinazione abitativa, ma la crisi e alcune difficoltà del consorzio edile hanno generato un paesaggio di attesa, fatto di scavi, recinzioni mobili, materiale costruttivo e terreni incolti.

Tre "scenografie di senso" per il testo pasoliniano, poetico e oscuro. Parole capaci di riverberare una luce diversa - arcaica, tragica - su quello che i nostri occhi colgono ora.

\section{L'lliade da levante a ponente}

Dopo quella dedicata al Pilade/Pasolini, in questa estate 2017 Archivio Zeta ha proposto una nuova maratona, dall'alba al tramonto: 1'Iliade/Poema della forza è stata portata, in quattro tempi, lungo l'asse della via Emilia a Bologna. Si tratta della sezione finale dell'opera omerica, quella che segue all'uccisione di Patroclo e quindi alla scelta di Achille di tornare in battaglia per vendicarsi. La creazione dello scudo di Achille, forgiato da Efesto per volontà di Teti, è stata messa in scena nel Bologna War Cemetery: di nuovo un cimitero di guerra, questa volta del Commonwealth. I bolognesi lo chiamano "il cimitero degli inglesi', ed è collocato accanto a quello, molto più esteso, che raccoglie le spoglie dei caduti polacchi: si trovano non a caso all'ingresso

Fig.5. Iliade/Poema della forza, Archivio Zeta al Cimitero del Commonwealth di Bologna, 2017 (foto: Massimo Marino).

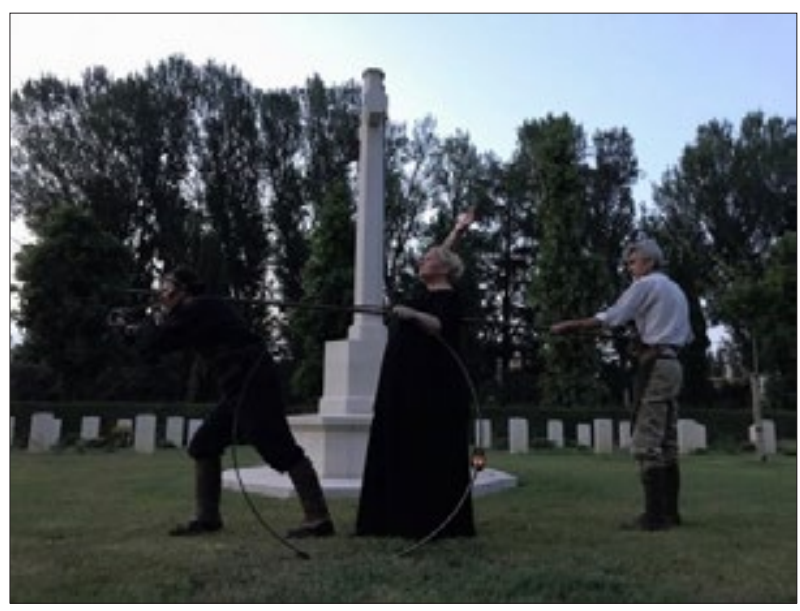


a est della città, poco oltre il fiume Savena. Da questo lato arrivarono, infatti, il 21 aprile 1945, i convogli alleati, per la battaglia che, insieme ai partigiani, portò alla liberazione di Bologna. Tra le tombe dei soldati, Enrica Sangiovanni ha interpretato la straordinaria ekphrasis dello scudo di Achille: la descrizione minuta e immaginifica di un oggetto meraviglioso e inesistente. Le armi divine per Achille, per fargli mietere vittime, sono forgiate nel luogo dove riposano soldati morti in battaglia.

Dopo qualche ora, l'incontro tra Achille e il fiume Scamandro, che scorre attorno a Troia, è stato rappresentato nei sotterranei dell'Ospedale Sant'Orsola: un luogo solitamente inaccessibile, di scorrimento veloce e invisibile, attraversato da tubature e mezzi di servizio. E qui dove il guerriero acheo fa strage, il fiume stremato dalla fatica di trascinare corpi di soldati uccisi si scaglia contro Achille. Dalla parte opposta della città, nel parco del lungo Reno, al tramonto Archivio Zeta ha messo in scena la morte di Ettore, la visita di Priamo ad Achille per riavere il corpo del figlio, il compianto e il rito funebre dell'eroe troiano, fino alla profezia di Cassandra dalle mura troiane.

Portando gli archetipi fondanti del pensiero tragico, da Omero a Eschilo, sui luoghi delle tragedie del Novecento, Archivio Zeta compie un lavoro di riflessione profonda sulla natura umana, tra mito e storia, poesia e cronaca. L'esperienza di un loro lavoro teatrale non ha nulla di rassicurante per il pubblico: non ha lo spazio contenente e limitato di una platea, né la comoda posizione seduta, né il buio che avvolge le reazioni più intime. Si partecipa a un'esperienza dura, percependo e partecipando alla fatica degli attori, del corpo a corpo con la parola che si libera in un paesaggio a sua volta gravato dalla violenza della storia e, in alcuni casi, del tempo presente.

\section{Risorse on line}

Dieter Oesterlen http://www.engramma.it/eOS2/index.php?id articolo=1944

Archivio Zeta

http://www.archiviozeta.eu/ 